\title{
Combined Laser Treatment in a Patient with Pigment Dispersion Secondary to a Large Iris Pigment Epithelial Cyst
}

\author{
Ümit Aykan'1, Özlem Yıldırım² \\ ${ }^{1}$ Department of Ophthalmology, Faculty of Medicine, Near East University, Nicosia, TRNC \\ ${ }^{2}$ Department of Ophthalmology, Faculty of Medicine, Mersin University, Mersin, Turkey
}

\begin{abstract}
We reported a case of bilateral extensive iris pigment epithelial cysts masquerading as pigment dispersion. A-30-year-old male patient presented with a dull pain in both eyes and a decreased visual acuity OD. He underwent a complete ophthalmic examination. OD was injected and the cells were graded as +3 and pigmented a +2 , in the OS. Intraocular pressures (IOP) were measured as 42 (OD) and 22 (OS) mmHg. Gonioscopy revealed a confluent accumulation of dense pigment in both eyes. Visual fields, peripapillary retinal nerve fiber layer thickness (Spectral OCT/SLO OTI-OPKO Health. Inc, Miami, FL) and optic nerve head tomography (HRT-II Heidelberg Engineering, Heidelberg, Germany) results were within normal limits. On ultrasound biomicroscopy (UBM), bilateral extensive cysts were identified in the midzonal portion of the iris and in the ciliary body. An, antiglaucomatous treatment was started. Then, we decided to perform both Nd:YAG laser iridocystotomyc and selective laser trabeculoplasty. Fourteen months after the combined therapy, the cysts had not recurred, and still apposed and the IOPs were under control without medication.
\end{abstract}

Key Word: Iris-ciliary body, pigment dispersion syndrome, laser iridocystotomy, laser trabeculoplasty, iris pigment epithelium cyst

Received: 12.05 .2012

Accepted: 04.06.2012

\section{Introduction}

Iris and ciliary body cysts are common, usually asymptomatic and sometimes clinically undetectable during the slit-lamp examination. Although they rarely have clinical significance, there may be some cases having significant angle compromise and must be treated as required for glaucoma (1-5). In some case reports, they were defined as a result of some medications or surgical procedures $(6,7)$.

The most common reason for referral of patients with iris pigment epithelial cyst (IPEC) and ciliary body cysts were one or more iris bulges or elevations, clinically visible cysts, narrow angles, increased intraocular pressure (IOP), or glaucoma and focal cataract (2). To the best of our knowledge, this is the first report on the effective laser treatment of bilateral, extensive IPEC masquerading as pigment dispersion (PD).

\section{Case Report}

A-30-year-old male patient presented with a dull pain (OU) a decreased visual acuity in OD (16/20). The OD was injected. There were moderate-intense pigmented cells in the anterior chambers of both eyes (OD +3 and $O S+2$ reaction). The pupils were semidilated and irregular without any sign of distortion. Peripheral iris transillumination was absent in both eyes. Gonioscopy (OU) revealed a confluent accumulation of dense pigment but there was no area of closure. Fundoscopy showed normal optic disc structure. IOP's were recorded as 42(OD) and 22(OS) $\mathrm{mmHg}$.
The visual fields, optic coherence tomography (Spectral OCT/SLO OTI-OPKO Health. Inc, Miami, FL) and Heidelberg Retinal Tomography-II (HRT-II Heidelberg Engineering, Heidelberg, Germany) results were all within normal limits. On ultrasound biomicroscopy (UBM) examination, extensive cysts were identified in the midzonal portion of the iris and in the ciliary body, OU (Figure 1, 2). The iris angiogram showed normal features without a focal or diffuse leakage pattern.

A diagnosis of ocular hypertension (OHT) due to pigmentary blockage of trabecular meshwork secondary to IPEC was made and maximal medical antiglaucomatous treatment was commenced to reduce the elevated IOP. The antiglaucomatous medications used were the timolol maleate+dorzolamide combination, twice daily, apraclonidine three times a day and acetazolamide orally $250 \mathrm{mg}$, three times a day. We did not use prostaglandin analogues due to their potential side effect in forming iris cysts (6). We decided to perform both Nd:YAG laser iridocystotomy (3 mj energy, 3 pulses per burst) in order to collapse the IPEC, thereby providing apposition and selective laser trabeculoplasty (SLT) (inferior $180^{\circ}, 0.7 \mathrm{~mJ}, 60$ shots) to enhance aqueous outflow and to lower IOP concurrently.

Immediately after laser therapy, there were no abrupt change in the IOP profile (either spike or dramatic decrease in IOP). A day after laser procedures the IOPs were under control but we still needed antiglaucomatous medications.

A day after the combined treatment, the midzonal IPECs were completely resorbed and both layers of pigment epithelium were apposed (Figure 3,4). The IOPs were under control with medications. The number of antiglaucomatous medica-

This study was presented as a poster presentation in European Glaucoma Society $9^{\text {th }}$ Congress, in Madrid, Spain, at September 12-17, 2010.

Address for Correspondence: Dr. Ümit Aykan, Department of Ophthalmology, Faculty of Medicine, Near East University, Nicosia, TRNC

Phone: +905323361810 E-mail: umitaykan@yahoo.com 


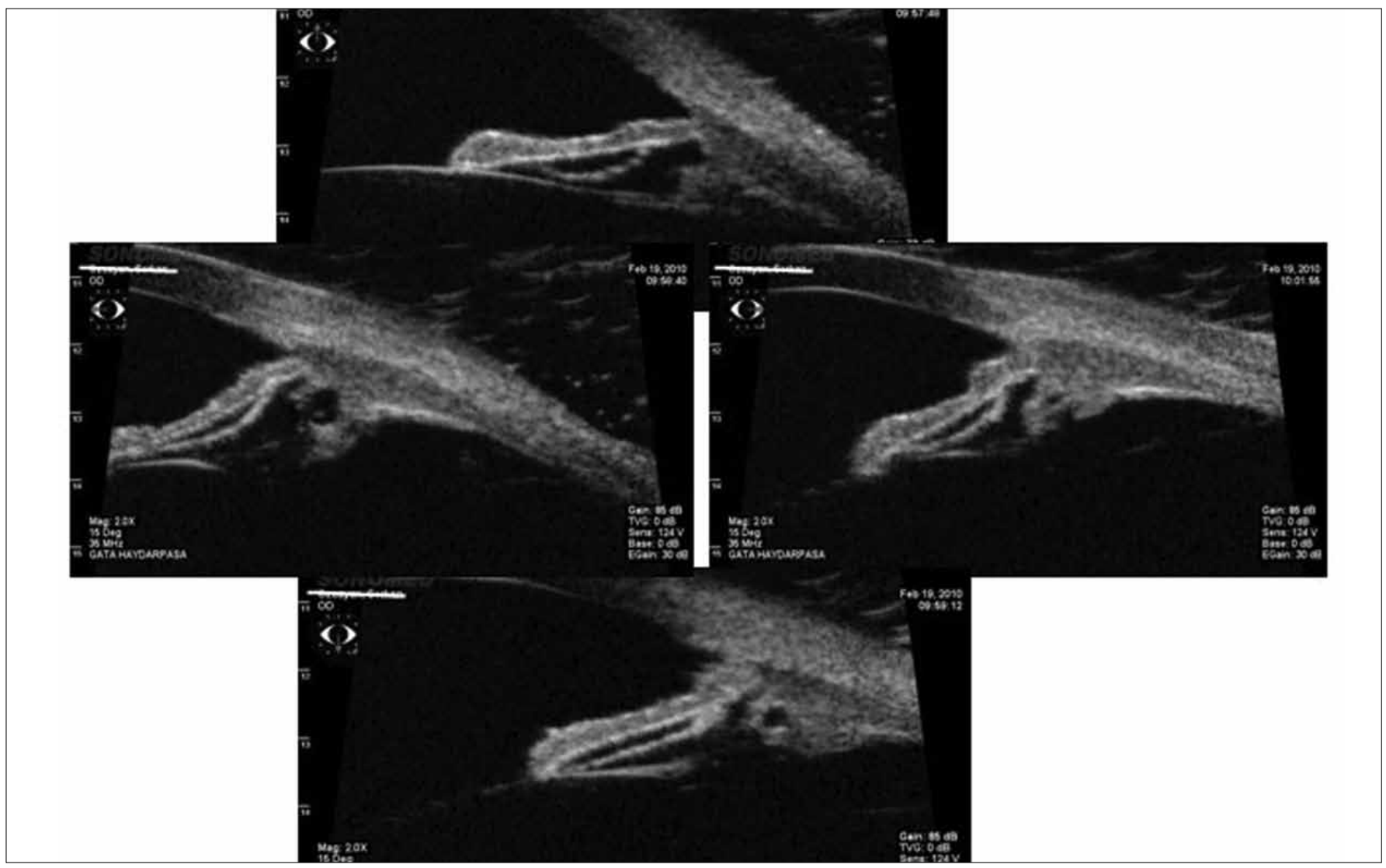

Figure 1. Ultrasound biomicroscopy images of four quadrants of the right eye before laser treatment

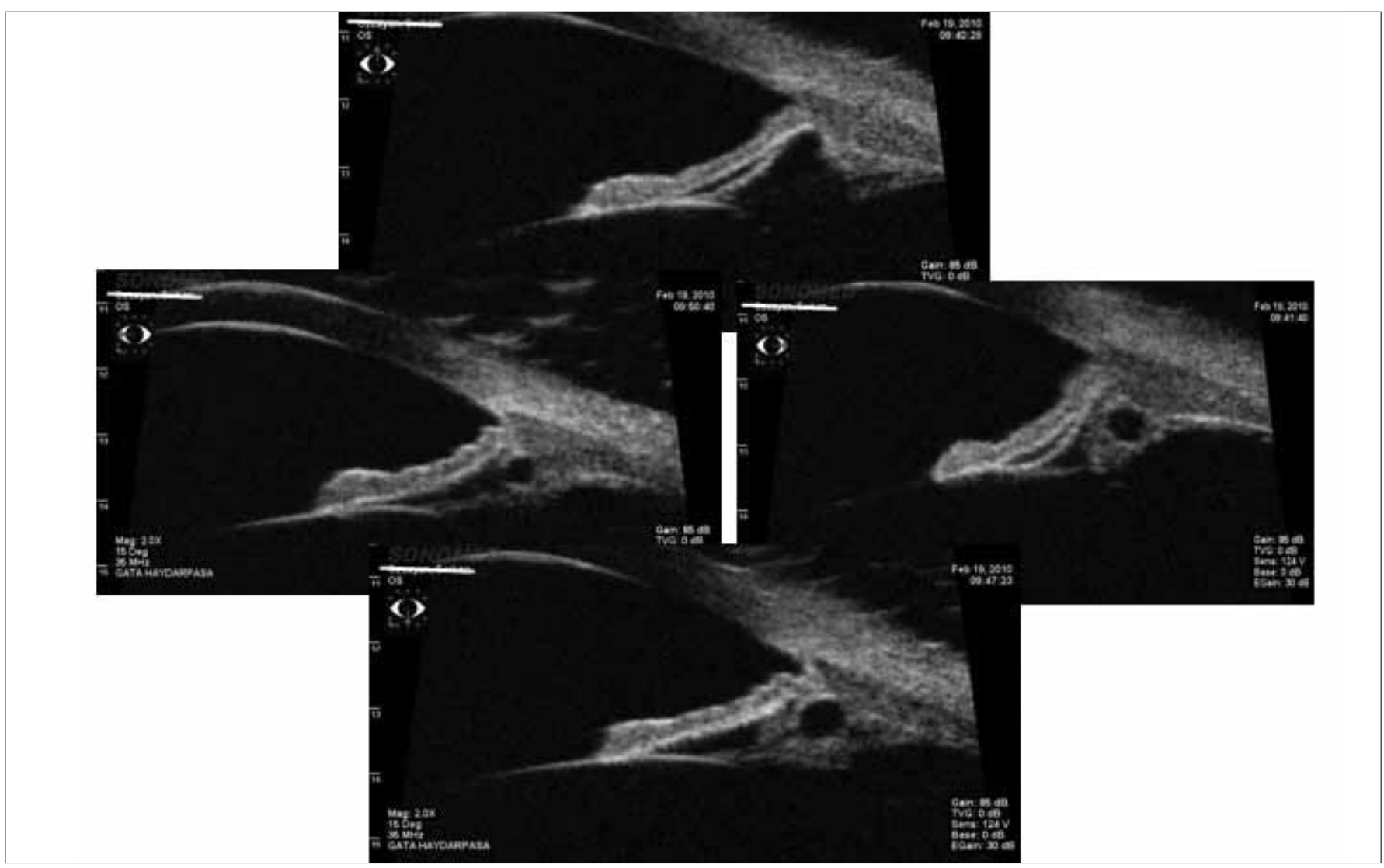

Figure 2. Ultrasound biomicroscopy images of four quadrants of the left eye before laser treatment 


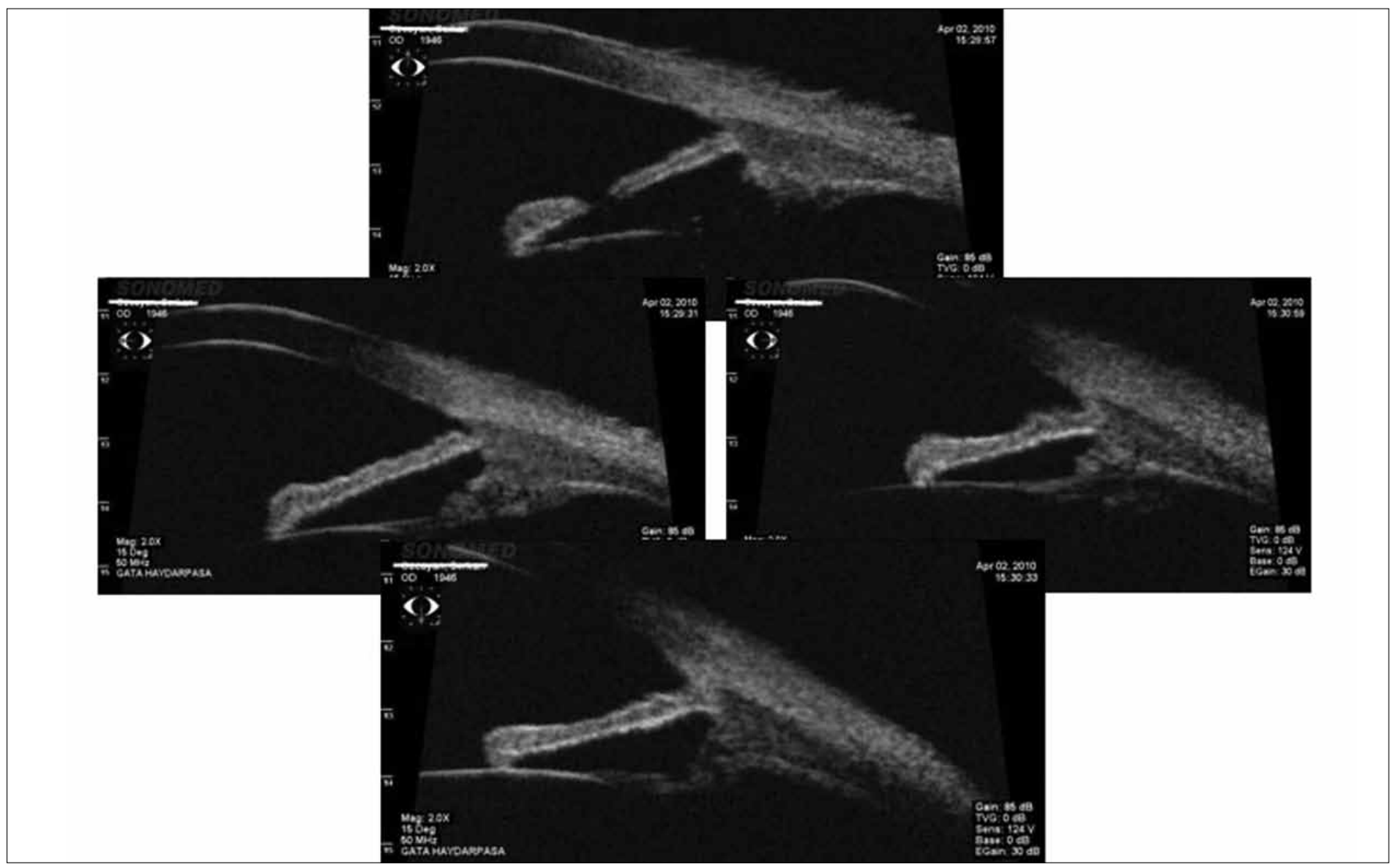

Figure 3. Ultrasound biomicroscopy images of four quadrants of the right eye after laser treatment

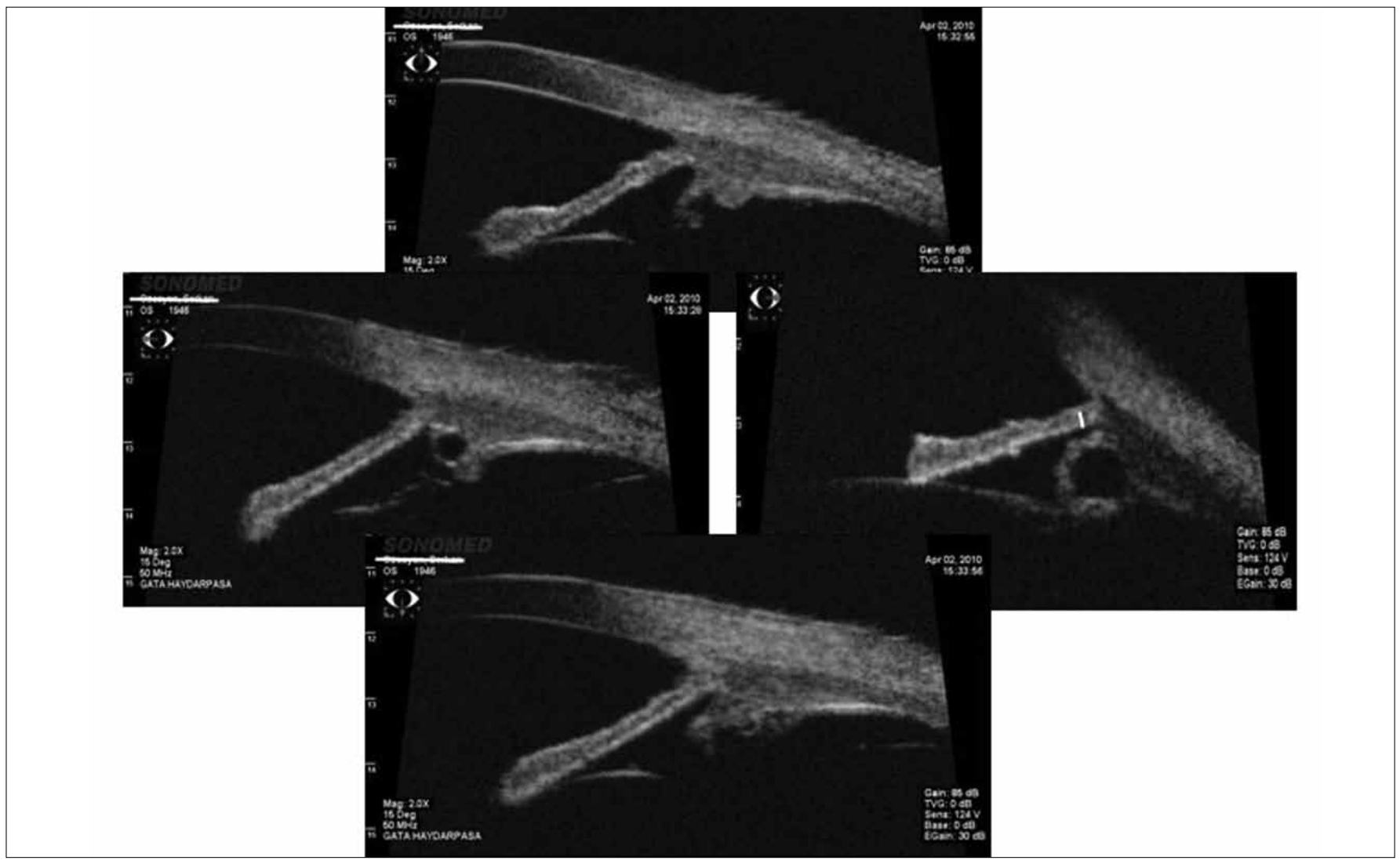

Figure 4. Ultrasound biomicroscopy images of four quadrants of the left eye after laser treatment 
tions used was decreased gradually and discontinued after 2 weeks. We observed that the pressures were within the normal range without medication during the follow-up period. We decided that SLT was efficient and safe in controlling the IOP.

Fourteen months after the combined therapy, the cysts had not recurred, and still apposed and the IOPs of both eyes were under control without medication.

\section{Discussion}

The patient described here who presented with PD secondary to IPEC which has been previously described in two different reports $(8,9)$. In these reports only the clinical course has been reported which was believed to be due to iris pigment but there was no information regarding the treatment of this condition.

In this unusual case, our treatment protocol was combined use of the laser iridocystotomy to provide the cyst resorption and SLT to enhance aqueous outflow thereby decreasing IOP.

It was of interest that the single application of laser iridocystotomy at the 12 o'clock position caused a complete cyst resorption in all quadrants of both eyes. During fourteen months of follow up, the cysts were always kept apposed.

Iris and ciliary body cysts can appear as different clinical presentations and significance. Iridociliary cysts are generally present in the irido-ciliary sulcus. They are thin walled and multiloculated. Glaucoma in patients with multiple irido-ciliary cysts is generally due to angle closure. Cysts can be collapsed locally by laser iridotomy, but generally this is not enough to open a significant amount of angle because of the multiloculated nature of the cysts.

In this case, there were both IPEC and ciliary body cysts. We believe that the IPEC was responsible for the clinical picture and the $C B$ cysts were silent lesions. The diagnosis of IPEC is apparent by the location under the iris and not in the ciliary sulcus. It is also apparent by the relatively thick wall which, as stated, was generally not the case with irido-ciliary cysts. Cysts of this type are less common and generally not associated with angle closure, as in this case with an open angle. This is essentially one large cyst. This is why a single iridotomy collapsed the entire cyst. It is important to make these distinctions in order not to propose that this treatment will be effective and safe for the iridociliary multi cystic angle closure variant.

The interesting finding here is the PD. It is possible that the extension of the iris pigment epithelium posteriorly produced PD by contact between the cyst wall and the zonule. The contact may induce a mild inflammatory reaction concurrently, along with the PD.

It is proposed that laser energy is absorbed in melanin granules in the trabeculum after SLT that triggers an inflam- matory reaction that effectively reduces IOP. There are some reports which clearly demonstrate the lack of effect of angle pigmentation on the IOP lowering results of SLT. In contradiction to those studies there were a few case reports of complications resulting from the use of SLT in heavily pigmented anterior chamber angles $(10,11)$. In our case, we provided a good IOP lowering effect in a short period after SLT procedure without any complications.

\section{Conclusion}

The UBM is a valuable tool for the diagnosis and following the evolution of these lesions. The combined use of laser iridocystotomy and SLT may be an effective treatment option draining the cyst, preventing further release of pigment and controlling the IOP by increasing the outflow of aqueous in patients with IPEC.

\section{Conflict of Interest}

No conflict of interest was declared by the authors.

\section{References}

1. McWhae JA, Rinke M, Crichton AC, Van Wyngaarden C. Multipl bilateral iridociliary cysts: ultrasound biomicroscopy and clinical characteristics. Can J Ophthalmol 2007;42:268-71. [CrossRef]

2. Lois N, Shields CL, Shields JA, Mercado G. Primary cysts of the iris pigment epithelium. Clinical features and natural course in 234 patients. Ophthalmology 1998;105:1879-85. [CrossRef]

3. Kuchenbecker J, Motschmann M, Schmitz K, Behrens-Baumann W. Laser iridocystotomy for bilateral acute angle-closure glaucoma secondary to iris cysts. Am J Ophthalmol 2000;129:391-3. [CrossRef]

4. Kathil P, Chin KJ, Ghaznawi N, Finger PT. Transpupillary Nd:YAG laser cystotomy for iris pigment epithelial cysts with secondary progressive angle closure. Ophthalmic Surg Lasers Imaging 2011;42:e40-3.

5. Honda N, Mimura T, Hotehama A, Usui T, Sugisaki K, Fukuoka S, et al. Laser treatment of giant iris cyst with nanophthalmos. Int Ophthalmol 2011;31:17-20. [CrossRef]

6. Pruthi S, Kashani S, Ruben S. Bilateral iris cyst secondary to topical latanoprost. Acta Ophthalmol 2008;86:233-4. [CrossRef]

7. Duan $X$, Zhang $Y$, Wang $N$. Laser treatment to large iris cyst secondary to trabeculectomy. Can J Ophthalmol 2007;42:316-7. [CrossRef]

8. Alward WL, Ossoinig KC. Pigment dispersion secondary to cysts of the iris pigment epithelium. Arch Ophthalmol 1995;113:1574-5. [CrossRef]

9. Chandler PA, Braconier HE. Spontaneous intra-epithelial cysts of iris and ciliary body with glaucoma. Am J Ophthalmol 1958;45:64-74.

10. Mcllraith I, Strasfeld M, Colev G, Hutnik CM. Selective Laser Trabeculoplasty as Initial and Adjunctive Treatment for Open-Angle Glaucoma. J Glaucoma 2006;15:124-30. [CrossRef]

11. Harasymowycz PJ, Papamatheakis DG, Latina M, De Leon M, Lesk MR, Damji KF. Selective laser trabeculoplasty (SLT) complicated by intraocular pressure elevation in eyes with heavily pigmented trabecular meshworks. Am J Ophthalmol 2005;139:1110-3. [CrossRef] 